\title{
Editorial: Frontiers in Silk Science and Technology
}

\author{
Nicola Maria Pugno ${ }^{1,2 *}$, Antonella Motta ${ }^{3,4}$ and David Kaplan ${ }^{5}$ \\ ${ }^{1}$ Laboratory of Bio-inspired, Bionic, Nano, Meta Materials \& Mechanics, Department of Civil, Environmental and Mechanical \\ Engineering, University of Trento, Trento, Italy, ${ }^{2}$ School of Engineering and Material Science, Queen Mary University of \\ London, London, United Kingdom, ${ }^{3}$ Department of Industrial Engineering and BIOtech Research Center, University of Trento, \\ Trento, Italy, ${ }^{4}$ European Institute of Excellence on Tissue Engineering and Regenerative Medicine, Trento, Italy, ${ }^{5}$ Department \\ of Biomedical Engineering, School of Engineering, Tufts University, Science and Technology Center, Medford, MA, \\ United States
}

Keywords: silk, spider, silkworm, mechanics, biomaterials, spiderweb

\section{Editorial on the Research Topic}

\section{Frontiers in Silk Science and Technology}

Silks from both spiders and silkworms have emerged as useful materials for addressing new challenges in materials science. These protein-based materials are often superior to many other polymers from a mechanical point of view and provide the possibility to realize composites with remarkable biological properties. Thus, studying raw silks, as well as silk-based natural and artificial composites, while controlling and manipulating processing conditions, is important to achieve advances in silk utility for materials science and technology. In doing this, a multidisciplinary and multiscale approach is crucial for studying silk and its derivatives.

This themed article collection focuses on some of the latest advances in silk science and technology, and topics include:

\section{OPEN ACCESS}

Edited and reviewed by: Gennady Mishuris,

Aberystwyth University, United Kingdom

*Correspondence: Nicola Maria Pugno nicola.pugno@unitn.it

Specialty section:

This article was submitted to

Mechanics of Materials,

a section of the journal

Frontiers in Materials

Received: 25 March 2021

Accepted: 20 April 2021

Published: 26 May 2021

Citation:

Pugno NM, Motta A and Kaplan D (2021) Editorial: Frontiers in Silk Science and Technology. Front. Mater. 8:685538. doi: 10.3389/fmats.2021.685538
- Protein structure and intermolecular interactions

- Characterization in the liquid and solid states

- Self-assembly in silk proteins

- Standardization of processing

- New uses for silks-e.g., optical and electronic materials

- Spider silk properties, uses, processing

- Recombinant silk

- Fabrication of bio-inspired materials with superior properties

- Silk from genetically modified organisms

- Silk mechanics

- Silk composites

- Silk bionanocomposites

This themed article collection features high-quality research from the 2019 edition of the Frontiers in Silk Sciences and Technologies conference (Trento, Italy), as well as significant contributions from experts worldwide working on the topic, across academia and industry, with a total of 17 contributions, as briefly summarized below reporting nearly verbatim the related abstracts.

In Riekel et al. the authors focused on "Nanoscale X-Ray Diffraction of Silk Fibers." This report focuses on current possibilities and perspectives of scanning X-ray nanodiffraction for probing nanoscale heterogeneities in silk fibers such as nanofibrils, skin-core morphologies, nanocrystalline inclusions and fine fibers down to submicron diameters.

In Pérez-Rigueiro et al. the authors focused on "Lessons From Spider and Silkworm Silk Guts." The results presented in previous work on the formation of fibers from silkworm and spider silk guts are reviewed and new information is included on the glands that allow the formation of 
these fibers. Silk gut fibers were obtained directly from the silk glands by immersion in a mild acid solution and subsequent stretching. The fibers produced were characterized in terms of their microstructure and mechanical properties. The comparison of silk gut fibers with their natural counterparts allows for new insights into processing, microstructure and properties of silkworm and spider silks.

In Sogawa et al. the authors focused on "Surface Analysis of Native Spider Draglines by FE-SEM and XPS." Although the physical and biological functions of the skin layer of spider dragline silk fibers have been studied and partially clarified, the morphology and elemental contents of these skin layer of silk fibers have not been investigated in detail. Here, the surface of Nephila clavata spider dragline was evaluated by field emission scanning electron microscopy (FE-SEM) and $\mathrm{X}$-ray photoelectron spectroscopy (XPS) to obtain surface morphological and molecular information. The FE-SEM images of the spider dragline indicated that it forms a bundle of microfibrils. This hierarchical structure induces faint fibrillar and network-like patterns on the surface of the dragline. XPS analysis revealed the presence of $\mathrm{Na}, \mathrm{P}$, and $\mathrm{S}$, which are reasonably explained considering the biological components of the major ampullate gland of spiders. The results will be important to consider in terms of the molecular transition of silk proteins to form hierarchical structures during spider dragline spinning.

In $\mathrm{Xu}$ et al. the authors focused on "Use of Silk Proteins to Form Organic, Flexible, Degradable Biosensors for Metabolite Monitoring." The development of sustainable and degradable biosensors and bioelectronics has implications for implantable systems, as well in addressing issues of electronic waste. Mechanically flexible and bioresorbable sensors can find applications at soft biological interfaces. While devices typically use metallic and synthetic components and interconnects that are non-degradable or have the potential to cause adverse tissue reactions, the use of nature-derived materials and conducting polymers can provide distinct advantages. In particular, silk fibroin and sericin can provide a unique palette of properties, providing both structural and functional elements. Here, a fully organic, mechanically flexible biosensor in an integrated 3electrode configuration is demonstrated. Silk sericin conducting ink is micropatterned on a silk fibroin substrate using a facile photolithographic process. Next, using a conducting polymer wire sheathed in silk fibroin, organic interconnects are used to form the electrical connections. This fully organic electrochemical system has competitive performance metrics for sensing in comparison to conventional systems, as verified by detection of a model analyte-ascorbic acid. The stability of the silk biosensor through biodegradation was observed, showing that the sensors can function for several days prior to failure. Such protein-based systems can provide a useful tool for biomonitoring of analytes in the body or the environment for controlled periods of time, followed by complete degradation, as transient systems for various applications.

In Veiga et al. the authors focused on "Recent Advances in Silk Sericin/Calcium Phosphate Biomaterials." Calcium phosphates (CaPs) have been widely used in the field of biomedical engineering as bone graft substitutes or as carriers for drug delivery applications. Recent developments have focused on combining $\mathrm{CaPs}$ with proteins to obtain functional biomaterials that accommodate a broader spectrum of functional requirements. Silk sericin was considered an unutilized protein by-product from the textile industry, generating tons of residues every year. However, much effort has been dedicated to its recovery after being associated with numerous biological properties such as antioxidant, antibacterial, anti-coagulation and regenerative activities. In the past years, sericin has also demonstrated to be suitable as a template for $\mathrm{CaP}$ mineralization. The present review focuses on the recent developments for the production of sericin/CaP composites, exploring their potential applications in bioengineering and opening new avenues in other research fields such as in the cosmetic, food and environmental sectors. In addition, this paper can also be useful as a guideline to design future research based on sericin/CaP biomaterials.

In Jeong et al. the authors focused on "Progress in Silk Fibroin Based Composite Scaffold/Hydrogel: Silk Fibroin/PEG Hydrogel for the RPE Regeneration a Promising Biomaterial for Clinical Application." Retinal pigment epithelium (RPE) plays a decisive role in the normal function of the retina, especially in the maintenance of photoreceptors. RPE dysfunction, loss of sight, and degeneration has been implicated as the cause of many retinal diseases including pigmented retinitis and age-related macular degeneration (AMD). Silk fibroin (SF) is a biodegradable natural polymer with biocompatibility, nontoxic, and non-immunological properties. In this study, hydrogel material was prepared by mixing it with PEG [poly (ethylene glycol)] a synthetic polymer. SF hydrogel (SH) and with PEG (SPH) were prepared with different sonication times and were physio chemically characterized by SEM, FTIR, compressive strength, porosity. In addition in vitro cytocompatibility was analyzed. As a result, the 20s SPH hydrogel exhibited superior cytocompatibility, cell adhesion, and improved cell growth compared to pure $\mathrm{SH}$. Their respective genes expression for retinal function and matrix production was also positively influenced by 20s SPH with an increase in gene expression of RPE65, CRALBP. The obtained results suggest that the 20s SPH hydrogel can be used as an alternative material for the application of retinal regeneration and delivery.

In Lopes et al. the authors focused on "Phase Diagram and Estimation of Flory-Huggins Parameter of Interaction of Silk Fibroin/Sodium Alginate Blends." Silk fibroin (SF) and sodium alginate $(\mathrm{SA})$ are natural polymers used to produce biomaterials. One of the strategies to improve the properties of these products is to prepare blends, which are partially miscible. Phase separation is observed, therefore, the thermodynamic analysis of this system is important to predict the final state and composition of this blends. This study explored blends with a different initial composition of SF, SA, and water (WA) at $25^{\circ} \mathrm{C}$ and neutral $\mathrm{pH}$. After phase separation, two phases were identified, one rich in SF and other rich in SA. The Flory-Huggins parameters of interaction of polymer-solvent and polymer-polymer were estimated using the extended equation and data of phase equilibrium, their values indicates the partial miscibility of the polymers. 
In Blamires et al. the authors focused on "Spider Silk Biomimetics Programs to Inform the Development of New Wearable Technologies." Wearable fabrics are predominantly produced from synthetic polymer fibers derived from petrochemicals. These have negative effects on the natural environment as a consequence of the manufacturing process, insurmountable waste production, and persistence of the fibers in ecosystems. With the use of wearables worldwide set to increase exponentially, more environmentally friendly fibers are sought. Natural fibers such as spider silk are produced using proteins in a water solvent, yet they have many superior qualities to synthetic fibers. Moreover, spiders can tune their silk properties as their ecological circumstances demand it. Research focused on the biomimetic potential of spider silks with an eye on the development of smart wearable fibers is accordingly a potentially lucrative area of research. There are nonetheless major challenges associated, including recovering the original mechanical performance within the fibers developed, scaling up production, keeping the production costs of the silk building blocks to a minimum, elucidating, and understanding the different silk genome sequences, and creating precision artificial spinning processes. The authors outline herein a template for a working framework for a spider silk biomimetics program that can inform designers and biological researchers alike. It suggests that an objective-focused research program utilizing a crossdisciplinary toolbox of top-down and bottom-up techniques is required. They close by providing some speculative examples stemming from current activities in their laboratories.

In Ferreira et al. the authors focused on "3D Structure and Mechanics of Silk Sponge Scaffolds Is Governed by Larger Pore Sizes." Three-dimensional scaffolds play an essential role in tissue engineering. Although essential, the tunability of the 3D scaffolds mechanical and transport properties remains a challenge. In this work, the authors present new approaches to advance the field. First, they applied their progressive $\mathrm{pH}$ acidification to mimic the natural silk gelation process before ice-templating ( -20 and $-80^{\circ} \mathrm{C}$ ); second, they fitted the mechanical properties using a connectivity model; third, they fitted the scaffolds mechanical relaxation to understand the transport properties; and fourth they used micro-CT to correlate the process parameters to the scaffolds' performances. Their results suggested that the free shrinkage of the scaffolds determined their final properties. They found, however, that the porosity (above 90\%) was anisotropic, similarly the tortuosity (between 1 and 1.3). They identified two major pore dimensions, the first one between 10 and $20 \mu \mathrm{m}$, and the second between 50 and $130 \mu \mathrm{m}$. Mechanically, their model suggested that the bulk modulus captured the elastic contribution and was controlled predominantly by the silk concentration. They tentatively associated the fractional modulus 1 to the collapse of the larger pores structures and was controlled mostly by the process temperature. They assigned the slow relaxation to the transport of fluid in the silk sponge scaffolds; and the fast relaxation with a viscoelastic relaxation. The silk concentration and process temperatures did not influence the latter. Overall, their use of the tomography, mechanical test, and detailed statistical analysis provides inroads into the interplay between process parameters (silk concentration and process temperature) and the multiple responses of the silk sponge scaffolds. The development of a new mechanical fitting for the compression test helped capture simply the different failure modes in the sponge scaffolds as well as correlating those events to relaxation and eventually transport properties.

In Silva et al. the authors focused on "Green Pathway for Processing Non-mulberry Antheraea pernyi Silk Fibroin/ChitinBased Sponges: Biophysical and Biochemical Characterization.” Silk protein fibroin (SF)-based matrices from non-mulberry, and mulberry silkworms are used for different applications in regenerative medicine. Silk fiber spun by the wild non-mulberry silkworm Antheraea pernyi (Ap) is also a promising biomedical material, due to the presence of the inherent tripeptide sequence of Arginine-Glycine-Aspartic acid (RGD) on the protein fibroin sequences. However, SF derived from the Ap cocoons still lacks exploitation in the healthcare field due to its poor solubility in the conventional solvents. This work addresses the application of green chemistry principles, namely the use of ionic liquids (ILs, 1-butyl-imidazolium acetate) and renewable resources such as Antheraea pernyi silk fibroin (ApSF) and chitin (Ch), for the fabrication of sponges from the blends of ApSF and Ch (APC). The formation of $\beta$-sheet in different contents during ApSF/Ch/IL was acquired by exposing gels to methanol/water and ethanol/water. The sponges were then obtained by freezedrying. This approach promotes the formation of both stable and ordered ApSF/Ch-based sponges. The developed sponges show suitable porosity and interconnectivity, appreciable swelling degree, and tuneable viscoelastic compressive properties for tissue engineering. Collectively, the structural properties of these ApSF/Ch-based sponges make them promising candidates for biomedical applications, namely cartilage regeneration.

In Brancato et al. the authors focused on "Tumor-Stroma Interactions Alter the Sensitivity of Drug in Breast Cancer." Flat cell cultures or xenografts are inadequate tools to unravel cancer complex biology. 3D in vitro tumor models garnered interest since they recapitulate better dynamic mechanisms of cancer, but a gold standard model that faithfully mimics solid cancer is not available yet. A 3D breast cancer model is fabricated using freeze-dried silk fibroin scaffolds. Breast cancer cell lines (MCF-7 and MDA-MB231) are seeded with normal mammary fibroblasts onto silk fibroin scaffolds ( 1 and $2 \mathrm{~mm}$ thick). Cell proliferation is monitored by Alamar blue assay, the $3 \mathrm{D}$ breast cancer model morphology is observed by confocal microscopy, and gene expression modulation concerning extracellular matrix markers is evaluated. Further, 3D bioengineered breast cancer models are treated with doxorubicin. Silk fibroin scaffolds allow the proliferation of cancer cells and fibroblasts. Cells growth is enhanced when cancer cells and fibroblasts are seeded together. Histological staining shows 3D cell organization. MMP-1, MMP2, MMP-3, Col-1, and fibronectin expression are upregulated in co-culture. After doxorubicin treatment, stronger reduction in cell activity is observed in $2 \mathrm{~mm}$ SF scaffold in comparison to $1 \mathrm{~mm}$. The $3 \mathrm{D}$ in vitro breast cancer model obtained can easily be scaled-up and translated to the preclinical testing of novel chemotherapeutics.

In Ribeiro et al. the authors focused on "Hierarchical HRPCrosslinked Silk Fibroin/ZnSr-TCP Scaffolds for Osteochondral 
Tissue Regeneration: Assessment of the Mechanical and Antibacterial Properties." The biomaterials requirements for osteochondral (OC) defects restoration simultaneously include adequate mechanical behavior, and the prevention of bacterial adherence and biofilm formation, without impairing local tissue integration. Bilayered and hierarchical scaffolds combining a cartilage-like layer interconnected to an underlying subchondral bone-like layer appeared as innovative technological solutions able to mimic the native OC tissue hierarchical architecture. This study is focused on the assessment of the combined compressionshear stresses and possible bacterial biofilm formation of hierarchical scaffolds prepared from a horseradish peroxidasecrosslinking reaction of silk fibroin (SF) combined with zinc $(\mathrm{Zn})$ and strontium (Sr)-doped $\beta$-tricalcium phosphate $(\beta$ TCP) for OC tissue regeneration. Scaffolds with undoped- $\beta$ TCP incorporation were used as control. Results showed that the bilayered scaffolds presented suitable aptitude to support compression and shear loading for OC tissue, with better mechanical properties for the $\mathrm{ZnSr}$-containing structures. Young and shear moduli presented values close to $0.01 \mathrm{MPa}$ in the region $10-20 \%$ strain. The investigation of biomaterials surface ability to prevent biofilm formation showed reduced bacterial adhesion of Escherichia coli (E. coli, gram-negative) and Staphylococcus aureus (S. aureus, gram-positive) on both scaffolds, thus suggesting that the proposed hierarchical scaffolds have a positive effect in preventing gram-positive and gramnegative bacteria proliferation.

In Pantano et al. the authors focused on "Doubling the Mechanical Properties of Spider Silk by C60 Supersonic Molecular Beam Epitaxy." Spider silk is one of the most fascinating natural materials, owing to its outstanding mechanical properties. In fact, it is able to combine usually self-excluding properties, like strength and toughness that synthetic fibers fail to replicate. Here, the authors report a method to further enhance the already excellent mechanical properties of spider's silk, producing nanocomposite fibers where the matrix of spider silk is reinforced with $\mathrm{C}_{60}$ molecules. These are deposited by Supersonic Molecular Beam Epitaxy (SuMBE) and are able to efficiently interact with silk, as evidenced by XPS analysis. As a consequence, upon proper adjustment of the fullerene kinetic energy, the treated fibers show improved strength, Young's modulus and toughness.

In Lee et al. the authors focused on "Recent Advances in Fluorescent Silk Fibroin." Silk is a natural macromolecular protein consisting of fibroin and sericin. Silk fibroin (SF), derived from Bombyx mori, is a representative fibrous protein that has been used mainly in fashion textiles and surgical sutures. Also, SF has been extensively applied as a potential biomaterial in a number of biomedical and biotechnological fields, as SF can be reconstituted in various forms through physical and chemical processes. In addition to direct use of SF with intrinsic structure and properties, there are many attempts to insert more novel functional properties into SF, retaining its favorable natural characteristics. In recent years, fluorescent silk obtained through various methods, such as the genetic modification or dye feeding, has been applied in a variety of medical fields. These functionalized silks have properties that can be applied to new and versatile fields such as drug delivery, and monitoring surgical and wound healing processes. This review focuses on the preparation methods and the latest technological advances on the use of fluorescent SF materials, especially their biomedical applications.

In Eom et al. the authors focused on "Multiscale Hybridization of Natural Silk-Nanocellulose Fibrous Composites With Exceptional Mechanical Properties." Because of the crisis of the petrochemical era, environmentally friendly natural polymers and related material processes are receiving great attention. Cellulose and silk are typical fibrous materials that consist of polysaccharides and proteins, respectively, and have excellent mechanical properties and high physicochemical stabilities due to their unique self-assembly-based hierarchical structures. In this study, highly stable high-strength silk fiber (HSF)-cellulose nanofibril (CNF) composites were prepared by the hierarchical fusion of micrometer-scale HSFs and nanometer-scale CNFs. This manufacturing process is cost-effective because the raw materials (HSFs and CNFs) are used as is. It is an eco-friendly process because it does not require the use of organic solvents or toxic reagents. In addition, it is an energy-efficient process because heat fusion $\left(120^{\circ} \mathrm{C}\right)$ takes only $10 \mathrm{~min}$. The results of the Direct Red 80 staining experiments confirm that up to 15 wt.\% CNFs were added to the HSF nonwoven. With the increase in the CNF amount, the nanometer-scale CNFs form a coating on the micro-scale HSFs. At the same time, the CNFs form bonds with the HSFs and increase the interfibrillar bonding strength of the CNF-coated HSFs. Therefore, the mechanical properties of the HSF/CNF composite and its stability in the water environment rapidly increase with increasing CNF concentration. In the case of HSF/CNF15, the mechanical and impact strengths increase by 110 and 228\%, respectively, compared with HSF non-woven without CNF. In addition, as CNFs are introduced, hydrophobicity of the surface and bulk of the HSF/CNF composite can be impacted, thereby maintaining structural stability in the water environment. This eco-friendly $\mathrm{HSF} / \mathrm{CNF}$ composite can be widely used as reinforcement for fiber-reinforced plastics as well as for other applications in the fibrous composite industry.

In Puerta et al. the authors focused on "Effects of Chemical Post-treatments on Structural and Physicochemical Properties of Silk Fibroin Films Obtained From Silk Fibrous Waste." Silk fibroin (SF) is a protein polymer claimed to have outstanding potential for medical applications. However, because of the manufacturing process, materials from regenerated SF exhibit a higher percentage of amorphous structures. The amorphous structures cause the material to be water soluble and can significantly limit its applications in wet biological environments. In order to increase the amount of crystalline structures and decrease the water solubility of SF materials, post-treatment with alcohols is usually employed. SF can be obtained from silk fibrous wastes (SFW), usually discarded in silk textile processes. This represents an opportunity to produce materials with high added value from low-cost natural sources. In this study, SF was obtained from SFW, and films were made thereof followed by a post-treatment by immersion or in a saturated atmosphere of methanol $(\mathrm{MeOH})$ or ethanol $(\mathrm{EtOH})$, using different 
exposure times. The resulting films were analyzed according to crystallinity, the percentage of crystalline and amorphous structures, and thermal stability. Also, water absorption and weight loss in aqueous media were determined. The results showed a significant increase in crystalline structures in all treated samples, varying according to the type and time of exposure to post-treatment conducted. The highest increase was shown in the case of the post-treatment by immersion in $\mathrm{MeOH}$ for $1 \mathrm{~h}$, with a $23 \%$ increase over the untreated sample. This increase in crystallinity was reflected in an increase in the degradation temperature and a degradation rate of $5.3 \%$ on day 7. The possibility of tuning the degree of crystallinity, as well as thermal stability and aqueous integrity of thin films of SFW, can be applied to adjust these materials to the requirements of specific biomedical applications.

Finally, in Greco et al. the authors focused on "Strong and Tough Silk for Resilient Attachment Discs: The Mechanical Properties of Piriform Silk in the Spider Cupiennius salei (Keyserling, 1877)." Spiders are able to produce different types of silk with different mechanical and biological properties. Piriform silk is produced to secure spiders and their webs to surfaces by using a nano-fibril network embedded in a cementlike matrix. Despite their fundamental role, the mechanical properties and function of these anchorages are still poorly understood due to the practical difficulties in nano-fibril sample preparation, the complexity of the system, and the high variation of attachment disc structures. Here the authors estimated the mechanical properties of this nano-fibril silk and those of the whole silk membrane in the large wandering spider Cupiennius salei through a combination of nanoindentation and nanotensile techniques and with the support of a simple analytical model. The results highlight the mechanical properties of the piriform silk, facilitating the modeling of silk composite mechanics. This could inspire the design of more efficient bio-inspired adhesives and fabrics.

\section{AUTHOR CONTRIBUTIONS}

All authors listed have made a substantial, direct and intellectual contribution to the work, and approved it for publication.

Conflict of Interest: The authors declare that the research was conducted in the absence of any commercial or financial relationships that could be construed as a potential conflict of interest.

Copyright (C) 2021 Pugno, Motta and Kaplan. This is an open-access article distributed under the terms of the Creative Commons Attribution License (CC BY). The use, distribution or reproduction in other forums is permitted, provided the original author(s) and the copyright owner(s) are credited and that the original publication in this journal is cited, in accordance with accepted academic practice. No use, distribution or reproduction is permitted which does not comply with these terms. 